\title{
Association of Insulin Resistance with Lower Glomerular Filtration Rate and All-Cause Mortality in the Korean Elderly Population: A Community-Based Prospective Cohort Study
}

\author{
Hyo Jin Kim, ${ }^{1}$ Jiwon Ryu, ${ }^{2}$ Shin Young Ahn, ${ }^{2}$ Sejoong Kim, ${ }^{1,2}$ Soo Lim, ${ }^{1,2}$ \\ Ki Young $\mathrm{Na}^{1,2}$ Ki Woong Kim, ${ }^{3,4}$ Hak Chul Jang, ${ }^{1,2}$ Dong-Wan Chae ${ }^{1,2}$ and \\ Ho Jun Chin ${ }^{1,2}$ \\ ${ }^{1}$ Department of Internal Medicine, Seoul National University College of Medicine, Seoul, Korea \\ ${ }^{2}$ Department of Internal Medicine, Seoul National University Bundang Hospital, Seongnam-si, Gyeonggi-do, \\ Korea \\ ${ }^{3}$ Department of Neuropsychiatry, Seoul National University Bundang Hospital, Seongnam-si, Gyeonggi-do, Korea \\ ${ }^{4}$ Department of Psychiatry, Seoul National University College of Medicine, Seoul, Korea
}

Insulin resistance (IR) is a common metabolic disorder in chronic kidney disease patients. In the elderly population, it is not clear whether IR is associated with lower glomerular filtration rate (GFR) and mortality. Here, we analyzed the association between IR and GFR, all-cause mortality (ACM), or cardiovascular mortality (CVM) in the elderly population. This was a community-based prospective study. Participants were selected by random sampling from those aged $\geq 65$ years in Seongnam, a satellite city of Seoul, Korea. A total of 743 participants were analyzed. IR was calculated using the homeostasis model assessment of insulin resistance (HOMA-IR). The mean age was $76.4 \pm 9.3$ years. After multivariate analysis, HOMA-IR was negatively associated with GFR as a continuous variable (per $10 \mathrm{~mL} / \mathrm{min} / 1.73 \mathrm{~m}^{2}$ increase, $\beta=$ $-0.10, P=0.004)$. Analyzing GFR as a categorical variable, HOMA-IR was still higher those with GFR $<60 \mathrm{~mL} / \mathrm{min} / 1.73 \mathrm{~m}^{2}$ (GFR $<60$ vs. $60-89$ and $\geq 90 \mathrm{~mL} / \mathrm{min} / 1.73 \mathrm{~m}^{2}: 1.3$ vs. 1.2 and 1.1 , respectively; $P=0.025$ and 0.019 ) by covariance analysis. During the follow-up period of $62.9 \pm$ 16.9 months, there were $168(22.6 \%)$ cases of ACM and $47(6.3 \%)$ cases of CVM. Those in the third quintile HOMA-IR group showed the lowest ACM rate, compared with the other quintiles $(P<0.001)$. CVM was not significantly associated with HOMA-IR. In conclusion, a lower GFR is associated with higher IR in the elderly population. Moreover, the lowest or the highest levels of HOMA-IR are associated with higher mortality rates.

Keywords: chronic kidney disease; elderly; glomerular filtration rate; insulin resistance; mortality Tohoku J. Exp. Med., 2013 December, 231 (4), 271-279. (C) 2013 Tohoku University Medical Press

\section{Introduction}

Chronic kidney disease (CKD) is a major public health problem; the prevalence of CKD has increased in the Korean population (Kim et al. 2009) and worldwide (Zhang and Rothenbacher 2008). The proportions of elderly people in the Korean population and the prevalence of diabetes mellitus (DM) according to age are also increasing. Aging (Hemmelgarn et al. 2006; Zhang and Rothenbacher 2008) and DM (Humphrey et al. 1989) are well-known risk factors for CKD. Therefore, older adults show a higher prevalence of CKD (Hemmelgarn et al. 2006; Zhang and Rothenbacher 2008) and DM (Humphrey et al. 1989), and this is characterized by insulin resistance (IR) (Scheen
2005).

Several studies have shown that non-diabetic patients with mild renal dysfunction (Fliser et al. 1998) and low estimated glomerular filtration rates (GFR) have higher levels of IR (Chen et al. 2003). IR and concomitant hyperinsulinemia are associated with an increased prevalence of CKD (Chen et al. 2003). In contrast, some studies did not find that lower GFR was associated with higher IR in the general population (Mohteshamzadeh et al. 2009; Park et al. 2013). In a study in an adult population undergoing routine health check-ups, Park et al. (2013) found that decreased GFR was not directly related to increased IR, but that parameters of metabolic syndrome were associated with decreased renal function rather than increased IR. In the

Received September 17, 2013; revised and accepted November 7, 2013. Published online November 29, 2013; doi: 10.1620/tjem.231.271. Correspondence: Ho Jun Chin, M.D., Ph.D., 12303 Department of Internal Medicine, Seoul National University Bundang Hospital, Gumidong 300, Bundang-gu, Seongnam-si, Gyeonggi-do 463-707, Korea.

e-mail: mednep@snubh.org 
elderly population, it is not clear whether lower GFR is associated with higher IR, and whether there is a relationship between IR and mortality. One recently published clinical study has shown that hypomagnesemia may be related to IR and a risk factor for mortality in CKD patients (Van Laecke et al. 2013).

The euglycemic clamp method is regarded as the "gold standard" for measuring IR; however, the method is invasive, time-consuming, and costly (Wallace and Matthews 2002). The homeostasis model assessment of IR (HOMAIR) is a mathematical model that calculates values for insulin sensitivity. Estimates of IR made using the HOMA-IR index are well correlated with IR values determined using the euglycemic clamp method (Matthews et al. 1985).

Here, we analyzed the associations between HOMAIR index and GFR, all-cause mortality (ACM), and cardiovascular mortality (CVM) in non-diabetic communitydwelling Korean older adults.

\section{Materials and Methods}

Study subjects

The present study was conducted as part of the Korean Longitudinal Study on Health and Aging (KLoSHA) (Park et al. 2007). KLoSHA was designed as a population-based, prospective cohort study of health, aging, and common geriatric disease of Korean older adults aged 65 years and over in Seongnam, a satellite city of Seoul, Korea. The protocol of KLoSHA was approved by the Institutional Review Board of Seoul National University Bundang Hospital in 2005 and 2010, and followed the principles of the Declaration of Helsinki. KLoSHA began in August 2005. Seongnam, one of the largest suburbs of Seoul, had a total population of 931,019 on August 1, 2005, including $61,730(6.6 \%)$ people aged 65 years or older (Fig. 1). At first, a simple random sample $(n=1,118 ; 1.81 \%)$ was drawn from a roster of 61,730 people on August 1, 2005, and letters and telephone calls were used to invite these subjects to participate in the study. Of the 1,118 subjects invited, 698 agreed to participate in the baseline KLoSHA study. Second, all elderly people aged 85 years or older living in Seongnam (3,136 subjects) were also contacted by letter or telephone and invited to participate, and 302 per-

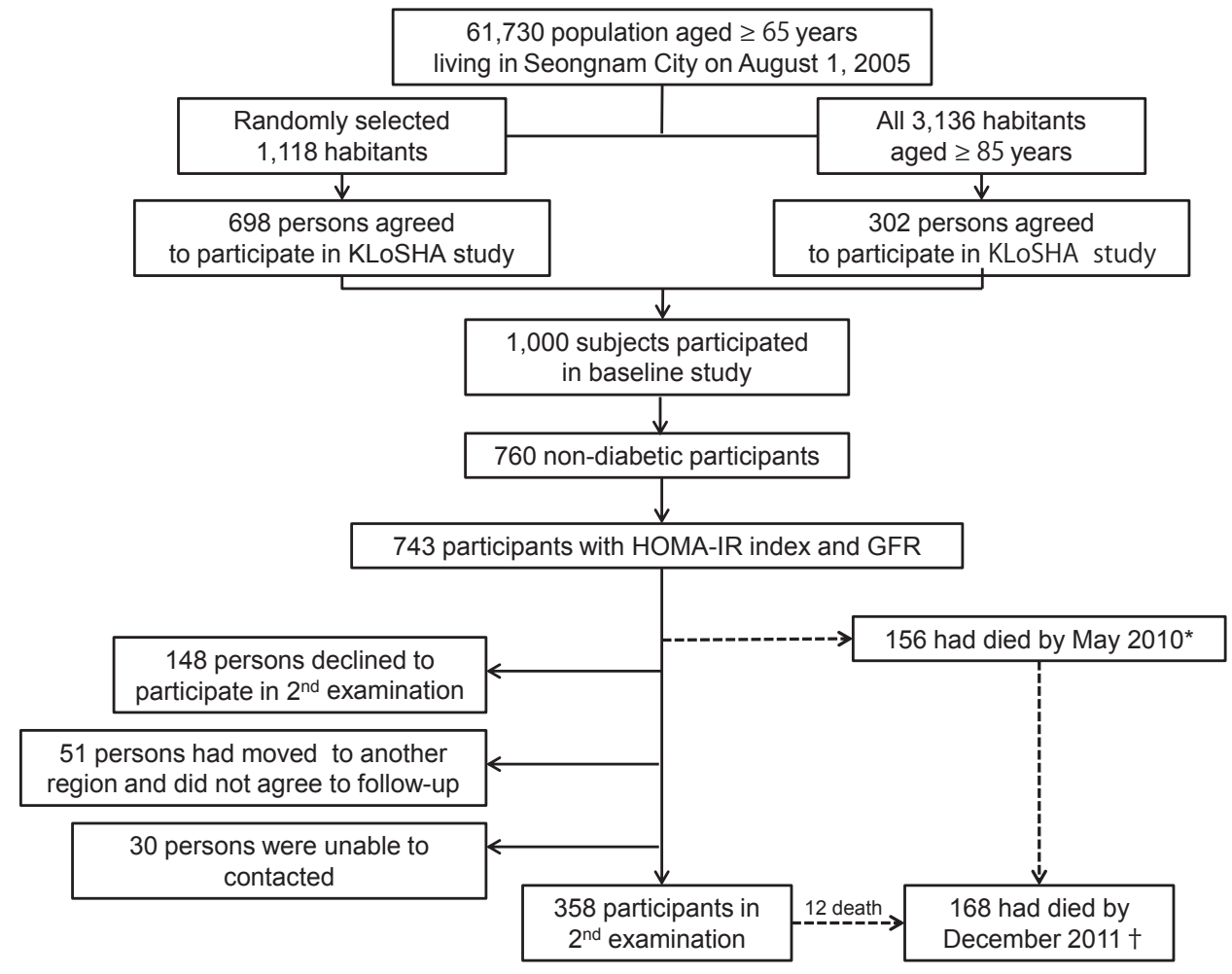

Fig. 1. Participants in the baseline and second examinations of the KLoSHA and their mortality during follow-up. KLoSHA was designed as a population-based, prospective cohort study of Korean older adults aged 65 years in Seongnam, a satellite city of Seoul, Korea. Seongnam had a total population of 931,019 on August 1, 2005, including 61,730 (6.6\%) people aged 65 years or older. At first, a simple random sample $(n=1,118 ; 1.81 \%)$ was drawn from a roster of 61,730 people on August 1, 2005. Of the 1,118 subjects invited, 698 agreed to participate in the baseline KLoSHA study. Second, all elderly people aged 85 years or older living in Seongnam $(3,136$ subjects $)$ were also contacted by letter or telephone and invited to participate, and 302 persons agreed to participate in the baseline KLoSHA study. Of a total of 1,000 subjects in the KLoSHA study, 760 persons did not have DM. Of them, 743 whose HOMA-IR and GFR levels were available were analyzed in this study. The follow-up study was conducted from May 2010 to December 2011.

*Mortality was determined by direct contact and through the Korea National Statistical Office (KNSO) database. $\dagger$ Mortality was identified through the KNSO database. KLoSHA, Korean Longitudinal Study on Health and Aging. 
sons agreed to participate in the baseline KLoSHA study. Of a total of 1,000 subjects in the KLoSHA study, 760 persons did not have DM. Of them, 743 whose HOMA-IR and GFR levels were available were analyzed in this study. In the initial study, all participants underwent a standardized clinical interview and laboratory testing. The follow-up study was conducted from May 2010 to December 2011. A total of $358(48.2 \%)$ subjects were followed-up, while $168(22.6 \%)$ had died, 148 (19.9\%) declined to participate in follow-up, 51 (6.9\%) had moved to another region, and $30(4.0 \%)$ were unable to be contacted. All evaluations were conducted at Seoul National University Bundang Hospital.

\section{Demographic and clinical data}

After an overnight fast of at least 12 hours' duration, subjects completed the questionnaires and underwent blood and urine tests. DM was defined as a reported history of DM, a plasma fasting glucose level of $\geq 126 \mathrm{mg} / \mathrm{dL}$, a serum hemoglobin A1c level $\geq 6.5 \%$, or the active use of an oral hypoglycemic agent. Hypertension was defined as systolic blood pressure of $140 \mathrm{mmHg}$ or greater, diastolic blood pressure of $90 \mathrm{mmHg}$ or greater, or the use of blood pressurelowering medication. Cardiovascular disease (CVD) history was defined as a self-reported history of exertional chest pain, angina pectoris, acute myocardial infarction, percutaneous coronary intervention or coronary artery bypass surgery, coronary artery endarterectomy, insertion of a pacemaker, congestive heart failure, or other cardiac operation. Proteinuria was defined as urine albumin trace or greater, determined by a dipstick urine test, and hematuria was defined as a urine red blood cell count of $\geq 5$ per high-power field under examination by light microscopy. Serum creatinine level was measured by the alkaline picrate Jaffe kinetic method using an automatic analyzer (Toshiba 200FR, Tokyo, Japan). Serum creatinine levels were calibrated to an assay traceable on an isotope dilution mass spectrometry (IDMS) device (Roche Diagnostics). Estimated GFR was calculated using the Chronic Kidney Disease Epidemiology (CKD-EPI) Collaboration equation (Levey et al. 2009). IR was assessed by HOMA-IR [fasting plasma glucose concentration $(\mathrm{mg} / \mathrm{L}) \times$ fasting plasma insulin $(\mu \mathrm{U} / \mathrm{mL}) / 405]$ index. Plasma glucose concentration was measured using the glucose oxidase method and a YSI 2300 STAT glucose analyzer (Yellow Spring Instrument Co., Yellow Springs, OH). Plasma insulin concentrations were measured using a radioimmunoassay (RIA) (Linco Research, Chesterfield, MO). The interassay coefficient of variation was $4 \%$.

\section{Outcome measurement}

We analyzed the correlation between HOMA-IR index and GFR levels, and the association between HOMA-IR index and ACM and CVM. GFR and HOMA-IR index values were analyzed as continuous and categorical variables. Participants were categorized into 3 GFR groups. The normal GFR group had a GFR $\geq 90 \mathrm{~mL} / \mathrm{min} / 1.73$ $\mathrm{m}^{2}$, the mild renal dysfunction group had a GFR $60-89 \mathrm{~mL} / \mathrm{min} / 1.73$ $\mathrm{m}^{2}$, and the moderate-to-severe renal dysfunction group had a GFR $<60 \mathrm{~mL} / \mathrm{min} / 1.73 \mathrm{~m}^{2}$. HOMA-IR index values were categorized into quintile groups. Data on ACM and CVM were obtained from the Korea National Statistical Office (KNSO) and by direct telephone contact.

\section{Statistical analysis}

Categorical variables were analyzed by $\chi^{2}$ test and presented as frequencies and percentages. Continuous variables were analyzed by one-way analysis of variance (ANOVA) and presented as mean \pm standard deviation. After Pearson's correlation coefficient testing, multivariate linear regression analysis was used to determine factors independently associated with HOMA-IR index. Adjusted HOMA-IR index values were derived and compared between GFR groups using covariance analysis (ANCOVA). The $\chi^{2}$ test was used to show frequency of mortality among groups and Kaplan-Meier method was used to analyze survival rate among groups as univariate analysis. We used Cox proportional hazards models to detect the independent risk factors related to mortality. $P$-values of $<0.05$ were considered statistically significant. SPSS Statistics software (SPSS version 19.0, Chicago, IL, USA) was used for statistical analysis.

\section{Results}

\section{Subjects characteristics}

Of the 1,000 subjects enrolled in the KLoSHA cohort, 743 non-diabetic participants with HOMA-IR index and GFR values available were included. The mean age was $76.4 \pm 9.3$ years, and $427(57.5 \%)$ subjects were female (Table 1). Hypertension and CVD were identified in $70.0 \%$ and $63.8 \%$ of participants, respectively. The mean HOMA-IR index value was $1.2 \pm 0.9$. The mean serum creatinine level was $0.9 \pm 0.3 \mathrm{mg} / \mathrm{dL}$ and the mean GFR value was $72.4 \pm 16.8 \mathrm{~mL} / \mathrm{min} / 1.73 \mathrm{~m}^{2}$.

\section{Clinical characteristics of the GFR groups}

There were 114 subjects in the normal GFR group, 451 subjects in the mild renal dysfunction group, and 178 subjects in the moderate-to-severe renal dysfunction group (Table 1). The participants in the moderate-to-severe renal dysfunction group were older $(P<0.001)$. The proportion of women was lower in the moderate-to-severe renal dysfunction group $(P=0.018)$. The prevalence of hypertension $(P=0.024)$ and $\operatorname{CVD}(P=0.025)$ increased with decreasing GFR level. Hemoglobin $(P<0.001)$ and highdensity lipoprotein cholesterol (HDL-C) $(P=0.002)$ levels were lowest in the moderate-to-severe renal dysfunction group. The frequency of proteinuria was highest in the moderate-to-severe renal dysfunction group $(P<0.001)$.

\section{Insulin resistance in the GFR groups and factors related to insulin resistance}

HOMA-IR index increased as GFR decreased, as shown by simple regression analysis $(r=-0.078, P=0.034)$. Linear regression analysis adjusted for age, gender, hypertension, hemoglobin, albumin, total cholesterol, and alanine aminotransferase (ALT) levels also showed a negative relationship between HOMA-IR index value and GFR as a continuous variable (per $10 \mathrm{~mL} / \mathrm{min} / 1.73 \mathrm{~m}^{2}$ change; $\beta=-0.10$, $P=0.004)$. Other factors, such as hemoglobin level $(\beta=$ $0.07, P=0.002)$, ALT level $(\beta=0.02, P<0.001)$, hypertension $(\beta=0.15, P=0.031)$, and gender (male vs. female, $\beta=$ $0.29, P<0.001)$ were also associated with HOMA-IR index values. When we estimated the HOMA-IR index values by ANCOVA adjusted for related factors, the moderate-tosevere renal dysfunction group had higher HOMA-IR val- 
Table 1. The clinical characteristics of participants according to GFR groups.

\begin{tabular}{|c|c|c|c|c|c|}
\hline & \multirow[b]{2}{*}{$\begin{array}{l}\text { All participants } \\
\quad(N=743)\end{array}$} & \multicolumn{4}{|c|}{ GFR groups } \\
\hline & & $\begin{array}{l}\text { Normal GFR } \\
\quad(n=114)\end{array}$ & $\begin{array}{l}\text { Mild renal } \\
\text { dysfunction } \\
(n=451)\end{array}$ & $\begin{array}{l}\text { Moderate-to-severe } \\
\text { renal dysfunction } \\
\quad(n=178)\end{array}$ & $P$-value \\
\hline Age (years) & $76.4 \pm 9.3$ & $69.5 \pm 5.7^{1}$ & $75.4 \pm 8.4^{2}$ & $83.2 \pm 8.9^{3}$ & $<0.001$ \\
\hline Gender (female \%) & 57.5 & 65.8 & 57.6 & 51.7 & 0.018 \\
\hline Hypertension (\%) & 70.0 & 63.2 & 69.4 & 75.8 & 0.024 \\
\hline CVD (\%) & 63.8 & 52.5 & 64.5 & 69.4 & 0.025 \\
\hline Smoking (never smoker \%) & 39.8 & 71.9 & 58.5 & 56.7 & 0.270 \\
\hline SBP (mmHg) & $131.1 \pm 17.6$ & $131.5 \pm 18.1$ & $131.1 \pm 16.8$ & $131.0 \pm 19.1$ & 0.971 \\
\hline DBP (mmHg) & $82.5 \pm 10.7$ & $83.0 \pm 10.0$ & $82.9 \pm 10.4$ & $81.2 \pm 11.8$ & 0.173 \\
\hline BMI $\left(\mathrm{g} / \mathrm{m}^{2}\right)$ & $23.5 \pm 3.2$ & $23.5 \pm 3.6$ & $23.7 \pm 3.2$ & $23.2 \pm 3.0$ & 0.337 \\
\hline Glucose $(\mathrm{mg} / \mathrm{dL})$ & $99.3 \pm 10.1$ & $99.2 \pm 10.3$ & $99.8 \pm 10.3$ & $98.1 \pm 9.5$ & 0.164 \\
\hline Insulin (uIU/ml) & $4.8 \pm 3.7$ & $4.5 \pm 2.4$ & $4.7 \pm 3.0$ & $5.2 \pm 5.5$ & 0.129 \\
\hline HOMA-IR & $1.2 \pm 0.9$ & $1.1 \pm 0.7$ & $1.2 \pm 0.8$ & $1.3 \pm 1.2$ & 0.236 \\
\hline Hemoglobin (g/dL) & $13.6 \pm 1.5$ & $13.5 \pm 1.4^{1,2}$ & $13.8 \pm 1.5^{2}$ & $13.2 \pm 1.5^{1}$ & $<0.001$ \\
\hline $\mathrm{CRP}(\mathrm{mg} / \mathrm{dL})$ & $0.2 \pm 0.7$ & $0.2 \pm 0.6$ & $0.2 \pm 0.6$ & $0.3 \pm 0.8$ & 0.366 \\
\hline Albumin (g/dL) & $4.1 \pm 0.3$ & $4.1 \pm 0.2^{1}$ & $4.1 \pm 0.3^{1,2}$ & $4.0 \pm 0.3^{2}$ & 0.025 \\
\hline Cholesterol (mg/dL) & $204.1 \pm 37.6$ & $209.0 \pm 38.1$ & $203.4 \pm 38.1$ & $202.7 \pm 35.6$ & 0.309 \\
\hline Triglyceride (mg/dL) & $131.0 \pm 80.1$ & $140.3 \pm 114.8$ & $129.7 \pm 76.8$ & $128.3 \pm 58.7$ & 0.391 \\
\hline HDL-C (mg/dL) & $60.6 \pm 15.5$ & $63.6 \pm 16.4^{1}$ & $61.1 \pm 15.7^{2}$ & $57.4 \pm 14.0^{2}$ & 0.002 \\
\hline Creatinine (mg/dL) & $0.9 \pm 0.3$ & $0.6 \pm 0.1^{1}$ & $0.8 \pm 0.2^{2}$ & $1.2 \pm 0.4^{3}$ & $<0.001$ \\
\hline $\operatorname{GFR}\left(\mathrm{ml} / \mathrm{min} / 1.73 \mathrm{~m}^{2}\right)$ & $72.4 \pm 16.8$ & $94.0 \pm 5.3^{1}$ & $76.2 \pm 8.3^{2}$ & $48.9 \pm 10.0^{3}$ & $<0.001$ \\
\hline Proteinuria $\geq$ trace $(\%)$ & 12.7 & 8.3 & 9.9 & 24.1 & $<0.001$ \\
\hline Hematuria (\%) & 11.7 & 8.3 & 12.4 & 13.8 & 0.191 \\
\hline
\end{tabular}

Normal GFR group: participants with GFR $\geq 90 \mathrm{ml} / \mathrm{min} / 1.73 \mathrm{~m}^{2}$, Mild renal dysfunction group: participants with GFR $60-89 \mathrm{ml} /$ $\min / 1.73 \mathrm{~m}^{2}$, Moderate-to-severe renal dysfunction group: participants with GFR $<60 \mathrm{ml} / \mathrm{min} / 1.73 \mathrm{~m}^{2}$, Number by the values: Tukey B, Dunnett T3 post hoc analysis in ANOVA, CVD, cardiovascular disease history - a history of exertional chest pain, angina pectoris, acute myocardial infarction, percutaneous coronary intervention or coronary artery bypass surgery, coronary artery endarterectomy, insertion of pacemaker, congestive heart failure, other cardiac operation; SBP, systolic blood pressure; DBP, diastolic blood pressure; BMI, body mass index; HOMA-IR, homeostatic model assessment of insulin resistance; CRP, C-reactive protein; HDL-C, high-density lipoprotein cholesterol; GFR, estimated glomerular filtration rate using CKD-EPI equation; Proteinuria, $\geq$ trace measured by urine dipstick test; Hematuria, $\geq 5$ high power field.

ues than the other 2 groups. The mean HOMA-IR index value in the moderate-to-severe renal dysfunction group (those with GFR $<60 \mathrm{~mL} / \mathrm{min} / 1.73 \mathrm{~m}^{2}$ ) was $1.3(95 \%$ confidence interval [CI], 1.20-1.46), while that in the mild renal dysfunction group (those with GFR $60-89 \mathrm{~mL} / \mathrm{min} / 1.73 \mathrm{~m}^{2}$ ) was $1.2(95 \% \mathrm{CI}, 1.08-1.23 ; P=0.025)$ and that in the normal GFR group (those with GFR $\geq 90 \mathrm{~mL} / \mathrm{min} / 1.73 \mathrm{~m}^{2}$ ) was $1.1(95 \%$ CI, 0.93-1.24; $P=0.019)$ (Fig. 2).

\section{All-cause mortality and cardiovascular mortality according to insulin resistance}

During the follow-up period of $62.9 \pm 16.9$ months, there were $168(22.6 \%)$ cases of ACM and $47(6.3 \%)$ cases of CVM. ACM rate was highest in the moderate-to-severe renal dysfunction group: normal GFR group, 10.5\% (12 subjects); mild renal dysfunction group, $18.4 \%$ (83 subjects); and moderate-to-severe renal dysfunction group, $41.0 \%$ (73 subjects) $(P<0.001$, Fig. 3A). When we analyzed survival rates by Kaplan-Meier method, ACM rate was also different among GFR groups ( $<60$ vs. $60-89$ and $\geq 90 \mathrm{~mL} / \mathrm{min} / 1.73 \mathrm{~m}^{2}: P<0.001$ and $<0.001$, respectively, Log-rank test) (data not shown). We divided the participants according to quintile level of HOMA-IR index. ACM rate differed according to HOMA-IR: first quintile group, $35.1 \%$ (52 subjects); second quintile group, $29.2 \%$ (42 subjects); third quintile group, $15.4 \%$ (23 subjects); fourth quintile group, $17.4 \%$ (26 subjects); and fifth quintile group, $16.9 \%$ ( 25 subjects $)(P<0.001$, Fig. 3B). When we analyzed survival rates by Kaplan-Meier method, ACM rate was also different among HOMA-IR quintiles $\left(1^{\text {st }}\right.$ vs. $2^{\text {nd }}$, $3^{\text {rd }}, 4^{\text {th }}$, and $5^{\text {th }}$ quintile: $P=0.253,<0.001,<0.001$, and $<0.001$, respectively, Log-rank test) (data not shown). In a Cox proportional hazards model adjusted for the factors related to mortality, the first, second, and fifth quintile groups had increased ACM rates compared to the third quintile group. The hazard ratio (HR) values were 2.925 (95\% CI, 1.69-5.09), 2.620 (95\% CI, 1.50-4.59), and 2.010 (95\% CI, 1.06-3.90), respectively (Table 2 ). The estimated 


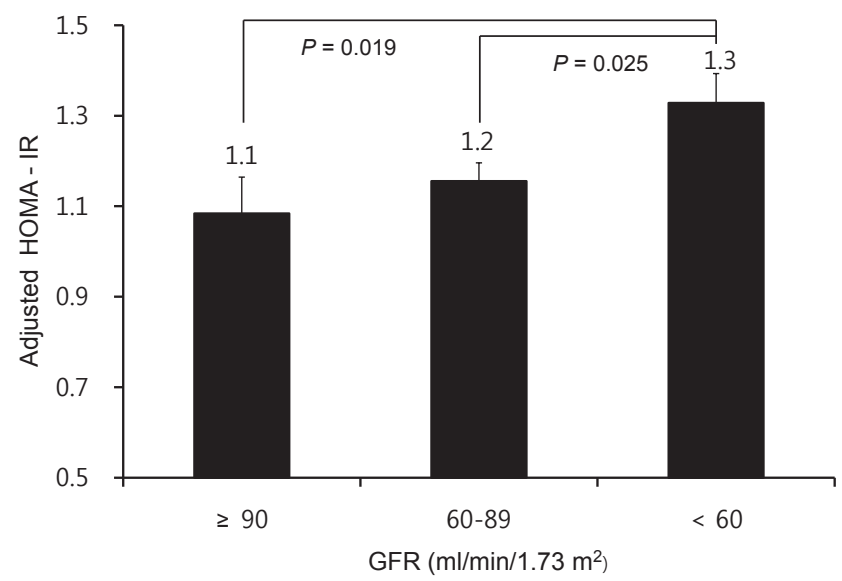

Fig. 2. Adjusted mean homeostasis model assessment of insulin resistance (HOMA-IR) index values.

Values were derived and compared among glomerular filtration rate groups with covariance analysis using the parameters that were associated with HOMA-IR index values. The moderate-to-severe renal dysfunction group had higher HOMA-IR values than the other 2 groups. The mean HOMA-IR index value in the moderate-tosevere renal dysfunction group (those with GFR $<60$ $\mathrm{mL} / \mathrm{min} / 1.73 \mathrm{~m}^{2}$ ) was 1.3 (95\% confidence interval [CI], $1.20-1.46)$, while that in the mild renal dysfunction group (those with GFR $\left.60-89 \mathrm{~mL} / \mathrm{min} / 1.73 \mathrm{~m}^{2}\right)$ was $1.2(95 \%$ CI, 1.08-1.23; $P=0.025)$ and that in the normal GFR group (those with GFR $\geq 90 \mathrm{~mL} / \mathrm{min} / 1.73 \mathrm{~m}^{2}$ ) was 1.1 (95\% CI, 0.93-1.24; $P=0.019)$. Error bars denote standard error.

5-year survival rates according to HOMA-IR group were $70.3 \%$ in the first quintile group, $73.2 \%$ in the second quintile group, $86.6 \%$ in the third quintile group, $85.2 \%$ in the fourth quintile group, and $76.5 \%$ in the fifth quintile group (Log-rank test, $P<0.001$ ). We searched for risk factors for ACM among the HOMA-IR quintile groups (Table 3). Participants in the first quintile HOMA-IR group were significantly older $(P<0.001)$ than those in the other quintile groups and had the lowest levels of total cholesterol $(P<$ $0.001)$, hemoglobin $(P<0.001)$, and body mass index (BMI) $(P<0.001)$. We also analyzed the interactions between HOMA-IR values and other risk factors associated with mortality. In the estimation of ACM, HOMA-IR index values interacted with age $(P$-interaction $=0.004)$, hemoglobin level $(P$-interaction $<0.001)$, total cholesterol level $(P$-interaction $<0.001)$, GFR $(P$-interaction $<0.001)$, and BMI $(P$-interaction $=0.042)$. However, HOMA-IR remained an independent risk factor for ACM after adjustment for these factors.

CVM rate significantly increased with decreasing GFR. The rate of CVM in the normal GFR group - was $3.5 \%$ (4 subjects), while the rates in the mild renal dysfunction group and the moderate-to-severe renal dysfunction group were $5.1 \%$ (23 subjects) and $11.2 \%$ (20 subjects), respectively $(P=0.026$, Fig. $3 \mathrm{C})$. When we analyzed survival rates by Kaplan-Meier method, CVM rate was also different among GFR groups ( $<60$ vs. $60-89$ and $\geq 90 \mathrm{~mL} /$ $\min / 1.73 \mathrm{~m}^{2}: P=0.005$ and 0.005 , respectively, Log-rank test) (data not shown). CVM rate also differed among the HOMA-IR quintile groups (Fig. 3D). CVM rate was 10.8\% (16 subjects) in the first quintile group, 7.4\% (11 subjects) in the second quintile group, $4.0 \%$ (6 subjects) in the third quintile group, $4.7 \%$ (7 subjects) in the fourth quintile group, and $4.7 \%$ (7 subjects) in the fifth quintile group ( $P=$ $0.019)$. When we analyzed survival rates by Kaplan-Meier method, CVM rate was also different among HOMA-IR quintiles $\left(1^{\text {st }}\right.$ vs. $2^{\text {nd }}, 3^{\text {rd }}, 4^{\text {th }}$, and $5^{\text {th }}$ quintile: $P=0.316$, $0.018,0.035$, and 0.040, respectively, Log-rank test) (data not shown). However, in a Cox proportional hazards model adjusted for age, C-reactive protein (CRP) level, HOMA-IR quintile group, hemoglobin level, and diastolic blood pressure, the independent parameters related to CVM were age (HR, 1.921; 95\% CI, 1.59-2.32; $P<0.001)$ and CRP level (HR, 1.314; 95\% CI, 1.08-1.59; $P=0.006$ ).

\section{Discussion}

We found that GFR was negatively correlated with HOMA-IR index values in non-diabetic Korean older adults. HOMA-IR quintile group was related to ACM risk, and the third quintile group showed the lowest incidence of ACM.

In younger adults, the association between low GFR and IR is controversial. Kobayashi et al. (2005) showed that the glucose disposal rate of 29 patients with CKD (mean age $56 \pm 14.6$ years) was significantly lower than that of healthy subjects. However, some studies did not find that lower GFR was associated with IR in younger adults. Johns et al. (2012) found that IR was not significantly associated with GFR in non-diabetic subjects aged 43-59 years. They explained that kidney dysfunction is related to components of metabolic syndrome, such as hypertension, rather than IR. Satirapoj et al. (2005) found that HOMA-IR was not dependent on GFR in non-diabetic adults. They determined that total body fat was an independent risk factor for IR. However, many studies have shown that GFR levels are negatively correlated with IR in younger adults. Similarly, in the elderly population, most studies have shown that GFR is negatively correlated with IR. Pham et al. (2012) demonstrated that a lower GFR was associated with degree of IR in subjects aged $\geq 65$ years. In that report, every $10 \mathrm{~mL} / \mathrm{min} / 1.73 \mathrm{~m}^{2}$ reduction in GFR was associated with a $2.2 \%$ increase in fasting insulin concentration and a $1.1 \%$ decrease in insulin sensitivity index. Among participants of the Health, Aging and Body Composition study (a study that included older individuals aged 70-79 years), kidney function was independently associated with IR (as defined by the highest quartile level of HOMA-IR) (Landau et al. 2011). There are no studies that have shown a lack of correlation between GFR and IR in the elderly population. Our findings of an association between lower GFR and degree of IR (as measured by HOMA-IR) are similar to previous results reported for the elderly population. 

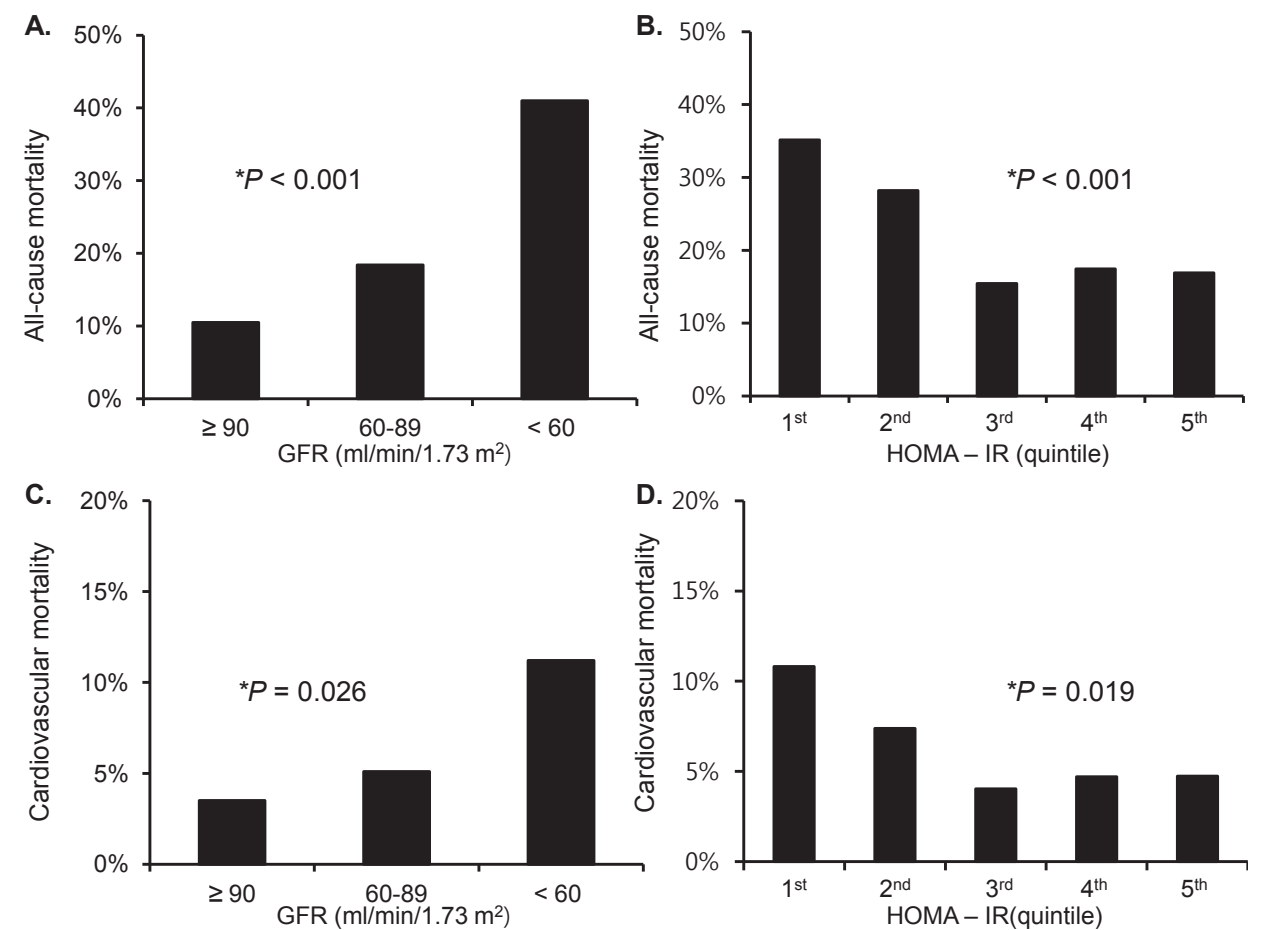

Fig. 3. ACM and CVM according to GFR groups and HOMA-IR quintiles.

A. Percentage of ACM according to GFR groups; B. Percentage of ACM according to HOMA-IR quintiles; C. Percentage of CVM according to GFR groups; D. Percentage of CVM according to HOMA- IR quintiles.

A. ACM rate was highest in the moderate-to-severe renal dysfunction group: normal GFR group, 10.5\% (12 subjects); mild renal dysfunction group, $18.4 \%$ (83 subjects); and moderate-to-severe renal dysfunction group, $41.0 \%$ (73 subjects) $\left(P<0.001, P\right.$-value indicates the differences among GFR groups by $\chi^{2}$ test as univariate analysis). B. ACM rate was lowest in the third quintile HOMA-IR group: first quintile group, 35.1\% (52 subjects); second quintile group, $29.2 \%$ (42 subjects); third quintile group, $15.4 \%$ (23 subjects); fourth quintile group, $17.4 \%$ (26 subjects); and fifth quintile group, $16.9 \%$ (25 subjects) $(P<0.001, P$-value indicates the differences among HOMA-IR quintile groups by $\chi^{2}$ test as univariate analysis). C. CVM rate was highest in the moderate-to-severe renal dysfunction group: normal GFR group, 3.5\% (4 subjects); mild renal dysfunction group, 5.1\% (23 subjects); and moderate-to-severe renal dysfunction group, $11.2 \%$ (20 subjects) $\left(P=0.026, P\right.$-value indicates the differences among GFR groups by $\chi^{2}$ test as univariate analysis). D. CVM rate was lowest in the third quintile HOMA-IR group: first quintile group, $10.8 \%$ (16 subjects); second quintile group, 7.4\% (11 subjects); third quintile group, 4.0\% (6 subjects); fourth quintile group, $4.7 \%$ (7 subjects); and fifth quintile group, $4.7 \%$ ( 7 subjects) $(P=0.019, P$-value indicates the differences among HOMA-IR quintile groups by $\chi^{2}$ test as univariate analysis). *All $P$-values indicate the differences among multiple columns in each panel. ACM, all-cause mortality; CVM, cardiovascular mortality; GFR, glomerular filtration rate; HOMA-IR, model assessment of insulin resistance.

IR is a known complication of end-stage renal disease (ESRD) and contributes to metabolic abnormalities in CKD, including uremic toxins, metabolic acidosis, and vitamin D deficiency (Mak 1998; Kielstein and Zoccali 2005). In addition, IR and CKD share a number of risk factors, such as obesity, poor diet, and sedentary lifestyle. It could be that these risk factors, along with vitamin D deficiency, are common in the aging and elderly population. Therefore, a decreased GFR is also correlated with degree of IR in the elderly population. Furthermore, development of IR occurs via multi-factorial mechanisms. Aging is associated with IR via changes in glucose tolerance, reduced insulin sensitivity and secretion, and increased peripheral insulin resistance (Broughton and Taylor 1991). In addition, reduced intake of carbohydrates, increased body weight and fat mass, and decreased fat-free mass can lead to IR in the elderly (Chen et al. 1988). In our cohort, participants in the first quintile of HOMA-IR values were older and had lower BMI values and total cholesterol levels. Obese subjects have greater IR than non-obese subjects (Goodpaster et al. 1997) and aging-related hyperlipidemia is associated with IR (Flannery et al. 2012). It is possible that lower BMI values and lower lipid levels could have affected HOMA-IR index values in our first HOMA-IR quintile group.

We investigated ACM and CVM to determine the impact of IR on outcomes. There are several reports of the relationship between IR, ACM, and CVM in the general population. Ausk et al. (2010) showed that HOMA-IR is associated with ACM (adjusted HR, 1.16 [95\% CI, 1.011.3] in a comparison of successive quartiles of HOMA-IR values in a linear model) in subjects with normal BMI $\left(<25.2 \mathrm{~kg} / \mathrm{m}^{2}\right.$, aged $\geq 17$ years $)$, and that CVM was significantly increased in the second, third, and fourth HOMA-IR quartiles compared with the lowest HOMA-IR quartile 
Table 2. Parameters included in the predictive equation for all-cause mortality estimated by Cox proportional hazards models.

\begin{tabular}{lccr}
\hline \multicolumn{1}{c}{ Variable } & HR & $95 \%$ CI & $P$-value \\
\hline Age (per 10 years) & 1.951 & $1.69-2.25$ & $<0.001$ \\
Hemoglobin (g/dL) & 0.871 & $0.78-0.97$ & 0.012 \\
HOMA-IR* & & & \\
$\quad 3^{\text {rd }}$ quintile & 1 & & \\
$1^{\text {st }}$ quintile & 2.925 & $1.69-5.09$ & $<0.001$ \\
$2^{\text {nd }}$ quintile & 2.620 & $1.50-4.59$ & 0.001 \\
$4^{\text {th }}$ quintile & 1.611 & $0.95-3.04$ & 0.141 \\
$5^{\text {th }}$ quintile & 2.010 & $1.06-3.90$ & 0.031 \\
GFR (per 10 $\left.\mathrm{ml} / \mathrm{min} / 1.73 \mathrm{~m}^{2}\right)$ & 0.875 & $0.80-0.96$ & 0.006 \\
\hline
\end{tabular}

The following variables were included to adjust by Cox proportional hazards models: age, total cholesterol, hemoglobin, GFR, HOMA-IR quintiles, proteinuria, C-reactive protein, and diastolic blood pressure.

$*$ HOMA-IR; $1^{\text {st }}$ quintile $\leq 0.67 ; 2^{\text {nd }}$ quintile $>0.67-0.85 ; 3^{\text {rd }}$ quintile $>0.85-1.07 ; 4^{\text {th }}$ quintile $>1.07-1.50 ; 5^{\text {th }}$ quintile $>1.50$.

HR, hazard ratio; CI, confidence interval; HOMA-IR, homeostatic model assessment of insulin resistance; GFR, estimated glomerular filtration rate.

Table 3. The characteristics of HOMA-IR group in aspects of risk factors to all-cause mortality.

\begin{tabular}{|c|c|c|c|c|c|c|c|}
\hline & \multirow{2}{*}{ Total (743) } & \multicolumn{6}{|c|}{ HOMA-IR Quintiles* } \\
\hline & & $1^{\text {st }}$ & $2^{\text {nd }}$ & $3^{\text {rd }}$ & $4^{\text {th }}$ & $5^{\text {th }}$ & $P$-value \\
\hline Age (years) & $76.4 \pm 9.3$ & $79.4 \pm 9.0$ & $77.7 \pm 9.5$ & $76.1 \pm 10.2$ & $74.6 \pm 8.5$ & $74.0 \pm 7.9$ & $<0.001$ \\
\hline Total cholesterol (mg/dL) & $204.1 \pm 37.6$ & $197.0 \pm 36.0$ & $194.3 \pm 34.2$ & $209.4 \pm 39.0$ & $207.4 \pm 38.1$ & $212.4 \pm 37.4$ & $<0.001$ \\
\hline Hemoglobin (g/dL) & $13.6 \pm 1.5$ & $13.1 \pm 1.5$ & $13.4 \pm 1.7$ & $13.7 \pm 1.4$ & $13.8 \pm 1.4$ & $14.0 \pm 1.3$ & $<0.001$ \\
\hline $\operatorname{BMI}\left(\mathrm{g} / \mathrm{m}^{2}\right)$ & $23.5 \pm 3.2$ & $21.3 \pm 3.0$ & $22.6 \pm 3.2$ & $23.3 \pm 2.5$ & $24.4 \pm 2.6$ & $25.8 \pm 2.9$ & $<0.001$ \\
\hline $\operatorname{GFR}\left(\mathrm{ml} / \mathrm{min} / 1.73 \mathrm{~m}^{2}\right)$ & $72.4 \pm 16.9$ & $75.8 \pm 16.3$ & $71.5 \pm 16.8$ & $71.8 \pm 16.3$ & $70.2 \pm 18.4$ & $72.7 \pm 15.9$ & 0.052 \\
\hline
\end{tabular}

$* H O M A-I R ; 1^{\text {st }} q u i n t i l e \leq 0.67 ; 2^{\text {nd }}$ quintile $>0.67-0.85 ; 3^{\text {rd }}$ quintile $>0.85-1.07 ; 4^{\text {th }}$ quintile $>1.07-1.50 ; 5^{\text {th }}$ quintile $>1.50$.

HOMA-IR, homeostatic model assessment of insulin resistance; BMI, body mass index; GFR, glomerular filtration rate.

group. Another study in patients with a mean age of $55.4 \pm$ 10.7 years showed that HOMA-IR was an independent predictor of CVM in patients with ERSD (adjusted HR, 4.24 [95\% CI, 1.83-11.55], top vs. lower 2 tertiles), but was not significantly associated with non-CVM (Shinohara et al. 2002). However, few studies have shown a correlation between IR and CVM or ACM in the elderly population. In our study, we showed that elderly subjects in not only the fifth quintile of HOMA-IR values but also the first quintile of HOMA-IR values had increased rates of ACM. We postulate that other factors could have influenced ACM in the first quintile HOMA-IR group. ACM increases with age in the elderly population. In addition, anemia, lower baseline hemoglobin levels, and hemoglobin decline are associated with increased mortality in the elderly population (Zakai et al. 2013). Zakai et al. (2005) showed that being in the lowest hemoglobin quintile was independently associated with mortality in an elderly cohort. In the general population, obesity is an important risk factor for mortality (Jee et al. 2006). However, this relationship is not evident in the elderly population: Janssen et al. (2005) showed that higher BMI, after adjustment for waist circumference, is associated with lower risk of mortality in older adults. They sug- gested that BMI could reflect a higher level of lean mass in subjects with the same waist circumference; lean mass was negatively correlated with ACM. Another study also showed that being underweight seems to be a stronger predictor of negative outcomes than obesity in older adults (Dey et al. 2001). BMI is not a reliable indicator of obesity; other factors, such as body composition and nutritional state, might be better predictors of mortality in the elderly population. Older age, lower baseline hemoglobin level, and lower BMI in the first quintile HOMA-IR group could have led to the increased rate of ACM in our elderly population. HOMA-IR had interactions with hemoglobin level, total cholesterol level, GFR, and BMI. Even though HOMA-IR is an independent risk factor for ACM after adjustment, we postulated that age, hemoglobin level, and BMI might have a greater effect on ACM than IR. In contrast to other studies in the general population, CVM was not correlated with HOMA-IR in our elderly population. We postulated that different baseline characteristics in our elderly population might have influenced the CVM results. Shinohara et al. (2002) showed that the highest HOMA-IR value (1.40 to 4.59 ) was an independent predictor of CVM, compared with the lower 2 tertiles (HOMA-IR values of 
0.28 to 1.39 ) in ESRD patients, suggesting that there is a threshold level of HOMA-IR in relation to CVM. Another study (Ausk et al. 2010) showed that those in the top 3 quartiles of IR (HOMA-IR > 1.4) had uniformly elevated CVM compared with those in the lowest quartile (HOMA-IR $\leq 1.4$ ). In our elderly population, HOMA-IR was relatively low, compared with values obtained in other studies. In addition, aging and high CRP levels are usually risk factors for CVM; however, participants in our fifth HOMA-IR quintile were younger, and CRP levels were similar in the 5 HOMR-IR groups. This could have influenced the CVM results in our study.

Our study has several strengths. Ours was a community-based prospective cohort study in the elderly population: the participants were representative of the elderly urban population. There are few studies of the association between GFR and IR in the elderly population, and therefore our study provides important data. Other strengths include the use of IDMS-traceable creatinine at a single institution (suitable for calculating GFR via the CKD-EPI equation) and the fact that ACM rates were surveyed by direct telephone contact as well as through the KNSO. However, our study had several limitations. We used the HOMA-IR index to estimate IR. The euglycemic clamp method is regarded as the "gold standard" for measuring IR; however, this method is invasive, time-consuming, and costly (DeFronzo et al. 1979; Wallace and Matthews 2002). Estimations of IR from the HOMA-IR index are well correlated with those obtained by the euglycemic clamp method (Matthews et al. 1985); therefore, we used the HOMA-IR index in this study. We calculated GFR via the CKD-EPI equation rather than through direct measurement. Even though the CKD-EPI equation has not been fully verified as a tool to estimate kidney function in the Korean population, the CKD-EPI equation is recommended by the Kidney Disease Improving Global Outcomes (KDIGO) guidelines (Levey et al. 2011). Also, many studies have shown that the CKD-EPI equation is more accurate than the Modification of Diet in Renal Disease (MDRD) Study equation (Murata et al. 2011; Earley et al. 2012), and a better predictor of ACM, CVM, and ESRD (Matsushita et al. 2012). Other limitations include the fact that CVD was investigated using data input according to diagnosis, which may have been subject to inaccuracies.

In conclusion, a lower GFR is associated with increased IR in the elderly Korean population. Moreover, elderly Korean people with the lowest and highest HOMA-IR values have higher mortality risk.

\section{Acknowledgements}

This study was supported by a grant from the Korean Health Technology R\&D Project, Ministry for Health, Welfare, \& Family Affairs, Republic of Korea (Grant No. A092077) and a grant for developing the Seongnam Health Promotion Program for the Elderly from Seongnam City Government in Korea (Grant No. 800-20050211).

\section{Conflict of Interest}

The authors have no financial conflict of interest.

\section{References}

Ausk, K.J., Boyko, E.J. \& Ioannou, G.N. (2010) Insulin resistance predicts mortality in nondiabetic individuals in the U.S. Diabetes Care, 33, 1179-1185.

Broughton, D.L. \& Taylor, R. (1991) Review: deterioration of glucose tolerance with age: the role of insulin resistance. Age Ageing, 20, 221-225.

Chen, J., Muntner, P., Hamm, L.L., Fonseca, V., Batuman, V., Whelton, P.K. \& He, J. (2003) Insulin resistance and risk of chronic kidney disease in nondiabetic US adults. J. Am. Soc. Nephrol., 14, 469-477.

Chen, M., Bergman, R.N. \& Porte, D. Jr. (1988) Insulin resistance and beta-cell dysfunction in aging: the importance of dietary carbohydrate. J. Clin. Endocrinol. Metab., 67, 951-957.

DeFronzo, R.A., Tobin, J.D. \& Andres, R. (1979) Glucose clamp technique: a method for quantifying insulin secretion and resistance. Am. J. Physiol., 237, E214-223.

Dey, D.K., Rothenberg, E., Sundh, V., Bosaeus, I. \& Steen, B. (2001) Body mass index, weight change and mortality in the elderly. A 15 y longitudinal population study of 70 y olds. Eur. J. Clin. Nutr., 55, 482-492.

Earley, A., Miskulin, D., Lamb, E.J., Levey, A.S. \& Uhlig, K. (2012) Estimating equations for glomerular filtration rate in the era of creatinine standardization: a systematic review. Ann. Intern. Med., 156, 785-795, W-270, W-271, W-272, W-273, W-274, W-275, W-276, W-277, W-278.

Flannery, C., Dufour, S., Rabol, R., Shulman, G.I. \& Petersen, K.F. (2012) Skeletal muscle insulin resistance promotes increased hepatic de novo lipogenesis, hyperlipidemia, and hepatic steatosis in the elderly. Diabetes, 61, 2711-2717.

Fliser, D., Pacini, G., Engelleiter, R., Kautzky-Willer, A., Prager, R., Franek, E. \& Ritz, E. (1998) Insulin resistance and hyperinsulinemia are already present in patients with incipient renal disease. Kidney Int., 53, 1343-1347.

Goodpaster, B.H., Thaete, F.L., Simoneau, J.A. \& Kelley, D.E. (1997) Subcutaneous abdominal fat and thigh muscle composition predict insulin sensitivity independently of visceral fat. Diabetes, 46, 1579-1585.

Hemmelgarn, B.R., Zhang, J., Manns, B.J., Tonelli, M., Larsen, E., Ghali, W.A., Southern, D.A., McLaughlin, K., Mortis, G. \& Culleton, B.F. (2006) Progression of kidney dysfunction in the community-dwelling elderly. Kidney Int., 69, 2155-2161.

Humphrey, L.L., Ballard, D.J., Frohnert, P.P., Chu, C.P., O'Fallon, W.M. \& Palumbo, P.J. (1989) Chronic renal failure in noninsulin-dependent diabetes mellitus. A population-based study in Rochester, Minnesota. Ann. Intern. Med., 111, 788-796.

Janssen, I., Katzmarzyk, P.T. \& Ross, R. (2005) Body mass index is inversely related to mortality in older people after adjustment for waist circumference. J. Am. Geriatr. Soc., 53, 21122118.

Jee, S.H., Sull, J.W., Park, J., Lee, S.Y., Ohrr, H., Guallar, E. \& Samet, J.M. (2006) Body-mass index and mortality in Korean men and women. N. Engl. J. Med., 355, 779-787.

Johns, B.R., Pao, A.C. \& Kim, S.H. (2012) Metabolic syndrome, insulin resistance and kidney function in non-diabetic individuals. Nephrol. Dial. Transplant., 27, 1410-1415.

Kielstein, J.T. \& Zoccali, C. (2005) Asymmetric dimethylarginine: a cardiovascular risk factor and a uremic toxin coming of age? Am. J. Kidney Dis., 46, 186-202.

Kim, S., Lim, C.S., Han, D.C., Kim, G.S., Chin, H.J., Kim, S.J., Cho, W.Y., Kim, Y.H. \& Kim, Y.S. (2009) The prevalence of chronic kidney disease (CKD) and the associated factors to CKD in urban Korea: a population-based cross-sectional 
epidemiologic study. J. Korean Med. Sci., 24 Suppl, S11-21.

Kobayashi, S., Maesato, K., Moriya, H., Ohtake, T. \& Ikeda, T. (2005) Insulin resistance in patients with chronic kidney disease. Am. J. Kidney Dis., 45, 275-280.

Landau, M., Kurella-Tamura, M., Shlipak, M.G., Kanaya, A., Strotmeyer, E., Koster, A., Satterfield, S., Simsonick, E.M., Goodpaster, B., Newman, A.B. \& Fried, L.F. (2011) Correlates of insulin resistance in older individuals with and without kidney disease. Nephrol. Dial. Transplant., 26, 2814-2819.

Levey, A.S., de Jong, P.E., Coresh, J., El Nahas, M., Astor, B.C., Matsushita, K., Gansevoort, R.T., Kasiske, B.L. \& Eckardt, K.U. (2011) The definition, classification, and prognosis of chronic kidney disease: a KDIGO Controversies Conference report. Kidney Int., 80, 17-28.

Levey, A.S., Stevens, L.A., Schmid, C.H., Zhang, Y.L., Castro, A.F. 3rd., Feldman, H.I., Kusek, J.W., Eggers, P., Van Lente, F., Greene, T. \& Coresh, J. (2009) A new equation to estimate glomerular filtration rate. Ann. Intern. Med., 150, 604-612.

Mak, R.H. (1998) Effect of metabolic acidosis on insulin action and secretion in uremia. Kidney Int., 54, 603-607.

Matsushita, K., Mahmoodi, B.K., Woodward, M., Emberson, J.R., Jafar, T.H., Jee, S.H., Polkinghorne, K.R., Shankar, A., Smith, D.H., Tonelli, M., Warnock, D.G., Wen, C.P., Coresh, J., Gansevoort, R.T., Hemmelgarn, B.R. \& Levey, A.S. (2012) Comparison of risk prediction using the CKD-EPI equation and the MDRD study equation for estimated glomerular filtration rate. JAMA, 307, 1941-1951.

Matthews, D.R., Hosker, J.P., Rudenski, A.S., Naylor, B.A., Treacher, D.F. \& Turner, R.C. (1985) Homeostasis model assessment: insulin resistance and beta-cell function from fasting plasma glucose and insulin concentrations in man. Diabetologia, 28, 412-419.

Mohteshamzadeh, M., Wong, C., Whiticar, R. \& Thomas, S. (2009) Is there a link between insulin resistance and chronic kidney disease in men with treated hypertension? Analysis of 5-year data. Am. J. Nephrol., 29, 116-122.

Murata, K., Baumann, N.A., Saenger, A.K., Larson, T.S., Rule, A.D. \& Lieske, J.C. (2011) Relative performance of the MDRD and CKD-EPI equations for estimating glomerular filtration rate among patients with varied clinical presentations. Clin. J. Am. Soc. Nephrol., 6, 1963-1972.

Park, J.H., Lim, S., Lim, J., Kim, K., Han, M., Yoon, I., Kim, J.M., Chang, Y.S., Chang, C., Chin, H.J., Choi, E.A., Lee, S.B.,
Park, Y.J., Paik, N.J., Kim, T., Jang, H.C. \& Kim, K.W. (2007) An overview of the Korean Longitudinal Study on Health and Aging. Psychiatr. Invest., 4, 84-95.

Park, J.H., Oh, S.W., Ahn, S.Y., Kim, S., Na, K.Y., Chae, D.W., Kim, S. \& Chin, H.J. (2013) Decreased estimated glomerular filtration rate is not directly related to increased insulin resistance. Diabetes Res. Clin. Pract., 99, 366-371.

Pham, H., Robinson-Cohen, C., Biggs, M.L., Ix, J.H., Mukamal, K.J., Fried, L.F., Kestenbaum, B., Siscovick, D.S. \& de Boer, I.H. (2012) Chronic kidney disease, insulin resistance, and incident diabetes in older adults. Clin. J. Am. Soc. Nephrol., 7, 588-594.

Satirapoj, B., Supasyndh, O., Boonyavarakul, A., Luesutthiviboon, L. \& Choovichian, P. (2005) The correlation of insulin resistance and renal function in non diabetic chronic kidney disease patients. J. Med. Assoc. Thai., 88 Suppl 3, S97-104.

Scheen, A.J. (2005) Diabetes mellitus in the elderly: insulin resistance and/or impaired insulin secretion? Diabetes Metab., 31 Spec No 2, 5S27-5S34.

Shinohara, K., Shoji, T., Emoto, M., Tahara, H., Koyama, H., Ishimura, E., Miki, T., Tabata, T. \& Nishizawa, Y. (2002) Insulin resistance as an independent predictor of cardiovascular mortality in patients with end-stage renal disease. J. Am. Soc. Nephrol., 13, 1894-1900.

Van Laecke, S., Nagler, E.V., Verbeke, F., Van Biesen, W. \& Vanholder, R. (2013) Hypomagnesemia and the Risk of Death and GFR decline in chronic kidney disease. Am. J. Med., 126, 825-831.

Wallace, T.M. \& Matthews, D.R. (2002) The assessment of insulin resistance in man. Diabet. Med., 19, 527-534.

Zakai, N.A., French, B., Arnold, A.M., Newman, A.B., Fried, L.F., Robbins, J., Chaves, P. \& Cushman, M. (2013) Hemoglobin decline, function, and mortality in the elderly: the cardiovascular health study. Am. J. Hematol., 88, 5-9.

Zakai, N.A., Katz, R., Hirsch, C., Shlipak, M.G., Chaves, P.H., Newman, A.B. \& Cushman, M. (2005) A prospective study of anemia status, hemoglobin concentration, and mortality in an elderly cohort: the Cardiovascular Health Study. Arch. Intern. Med., 165, 2214-2220.

Zhang, Q.L. \& Rothenbacher, D. (2008) Prevalence of chronic kidney disease in population-based studies: systematic review. BMC Public Health, 8, 117. 